\title{
Thermographic Imaging of the Space Shuttle during Re-Entry Using a Near Infrared Sensor
}

\author{
Joseph N. Zalameda ${ }^{* a}$, Thomas J. Horvath ${ }^{\mathrm{a}}$, Robbie V. Kerns ${ }^{\mathrm{a}}$, Eric R. Burke ${ }^{\mathrm{a}}$, Jeff C. Taylor ${ }^{\mathrm{b}}$, Tom \\ Spisz ${ }^{\mathrm{b}}$, David M. Gibson ${ }^{\mathrm{b}}$, Edward J. Shea ${ }^{\mathrm{c}}$, C. David Mercer ${ }^{\mathrm{d}}$, Richard J. Schwartz ${ }^{\mathrm{e}}$, Steve Tack ${ }^{\mathrm{f}}$, \\ Brett C. Bush ${ }^{\mathrm{g}}$, Ronald F. Dantowitz ${ }^{\text {, and Marek J. Kozubal }}{ }^{\mathrm{h}}$ \\ ${ }^{a}$ NASA Langley Research Center Hampton, VA 23681 \\ bohns Hopkins University Applied Physics Laboratory, Laurel, MD 20723 \\ ${ }^{\mathrm{C}}$ Futron Corporation, Hampton, VA 23666 \\ ${ }^{\text {d}}$ SGT NASA Langley Research Center Hampton, VA 23681 \\ eAMA NASA Langley Research Center Hampton, VA 23681 \\ ${ }^{\mathrm{f}}$ Naval Air Warfare Center Weapons Division, Pt. Mugu, CA 93042 \\ ${ }^{g}$ Raytheon/Photon Research Associates Inc., San Diego, CA 92121 \\ ${ }^{\mathrm{h}}$ Celestial Computing Inc., Jamaica Plain, MA. 02130
}

\begin{abstract}
High resolution calibrated near infrared (NIR) imagery of the Space Shuttle Orbiter was obtained during hypervelocity atmospheric re-entry of the STS-119, STS-125, STS-128, STS-131, STS-132, STS-133, and STS-134 missions. This data has provided information on the distribution of surface temperature and the state of the airflow over the windward surface of the Orbiter during descent. The thermal imagery complemented data collected with onboard surface thermocouple instrumentation. The spatially resolved global thermal measurements made during the Orbiter's hypersonic re-entry will provide critical flight data for reducing the uncertainty associated with present day ground-toflight extrapolation techniques and current state-of-the-art empirical boundary-layer transition or turbulent heating prediction methods. Laminar and turbulent flight data is critical for the validation of physics-based, semi-empirical boundary-layer transition prediction methods as well as stimulating the validation of laminar numerical chemistry models and the development of turbulence models supporting NASA's next-generation spacecraft. In this paper we provide details of the NIR imaging system used on both air and land-based imaging assets. The paper will discuss calibrations performed on the NIR imaging systems that permitted conversion of captured radiant intensity (counts) to temperature values. Image processing techniques are presented to analyze the NIR data for vignetting distortion, best resolution, and image sharpness.
\end{abstract}

Keywords: HYTHIRM, Space Shuttle thermography, hypersonic imaging, near infrared imaging, histogram analysis, singular value decomposition, eigenvalue image sharpness

\section{INTRODUCTION}

The NASA Langley Research Center led HYpersonic THermodynamic InfraRed Measurement (HYTHIRM) project culminated in the acquisition of high-resolution calibrated infrared imagery of the Space Shuttle Orbiter during hypersonic atmospheric entry. The thermal imaging provided a unique and never before observed perspective on the global distribution of surface temperature and the state of the airflow (i.e., laminar/turbulent) over the entire windward surface of the Orbiter during re-entry. Laminar and turbulent flight data are critical for the validation of physics-based, semi-empirical boundary-layer transition prediction methods, as well as stimulating the validation of laminar numerical chemistry models and the development of turbulence models supporting NASA's next-generation spacecraft [1]. This paper will provide details behind the use of a digital near infrared (NIR) imaging system and describe the characterizations and procedure performed to obtain quantitative temperature imagery. Past attempts by the Cast Glance aircraft to image the Orbiter during STS-121 and STS-115 missions have resulted in successful image acquisitions,

*joseph.n.zalameda@nasa.gov; phone 1 757-864-4793; fax 1 757-864-4914; http://researchtech.larc.nasa.gov/branches/nondes_eval_sci.htm 
STS-121 (July 2006)

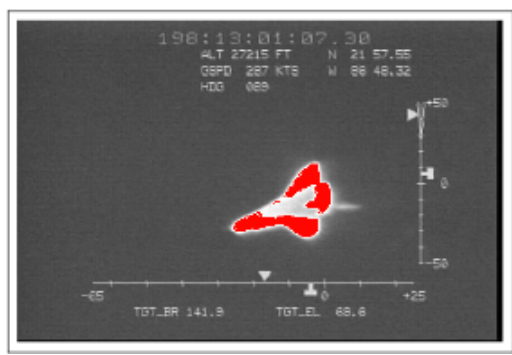

STS-115 (Sept. 2006)

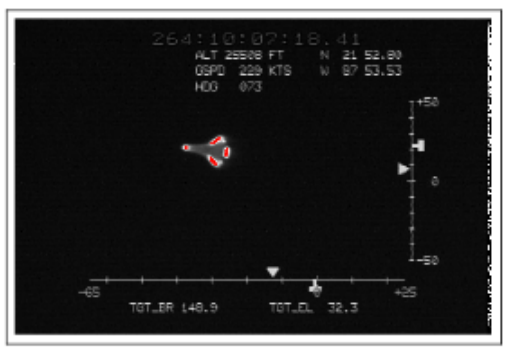

Figure 1. STS-121 and STS-115 images showing early promise of imaging during hypersonic re-entry.

however, the quality of the acquired data allowed for limited data processing [2-5]. During both missions, the Orbiter imagery was obtained using an analog-based NIR imaging system and due to image saturation, limited quantitative analysis could be performed. The STS-121 and STS-115 acquired images are shown in Figure 1 with pixels at nose, leading edges, and tail indicating saturation. The early results, however, indicated significant potential for imaging the Space Shuttle during hypersonic re-entry. As a result, a digital NIR imaging system was implemented after a series of rigorous testing at the Solar Tower Thermal Test Facility at Sandia National Laboratories [6,7]. Implementation of the digital NIR camera system has resulted in NIR imagery with improved dynamic range, instantaneous field of view, and signal to noise [8-11]. The characterizations performed on the digital NIR system included radiometric, spatial, and spectral measurements using blackbody radiation sources and known targets [12,13]. An assessment of the collected data, using the digital NIR imaging sensor, for STS-119, STS-125, STS-128, STS-131, STS-132, STS-133, and STS-134 missions, is presented. A majority of these missions contained a detailed test objective (DTO) to study boundary layer transition (BLT) [11]. The DTO BLT mission flew a modified tile with a protuberance to induce turbulent flow. This caused increased heating at the trip location and therefore required a high dynamic range NIR imaging system.

The data were collected using a US Navy NP-3D Orion aircraft (STS-119, STS-125, STS-128, STS 132, and STS 133) and a land based Mobil Aerospace Reconnaissance System (MARS) (STS-131 and STS-134). The imaging assets are shown in Figure 2. A typical data collect contained approximately 10,000 to 13,000 image frames (20 - 27 gigabytes of data). Image quality is influenced by sun angle, aircraft vibration, Orbiter orientation, distance from Orbiter to imager, atmospheric attenuation, and tracking jitter. Nevertheless, excellent imagery is obtained, by selecting or "cherry picking" the best images. A histogram method is used to analyze the thermal imagery to determine the images of interest [12]. The histogram analysis method determines image saturation, frame dropouts and signal loss due to vignetting distortion. In addition, a technique to measure image sharpness using image eigenvalue decomposition approach is shown to determine image sharpness [14,15]. Selected images are then converted to quantitative 2dimensional temperature images. The process used to convert the raw image counts to 2-dimensional temperature images is presented using atmospheric corrections [16,17]. Lastly, examples of HYTHIRM remotely measured temperatures are compared to the Orbiters' onboard thermocouples.
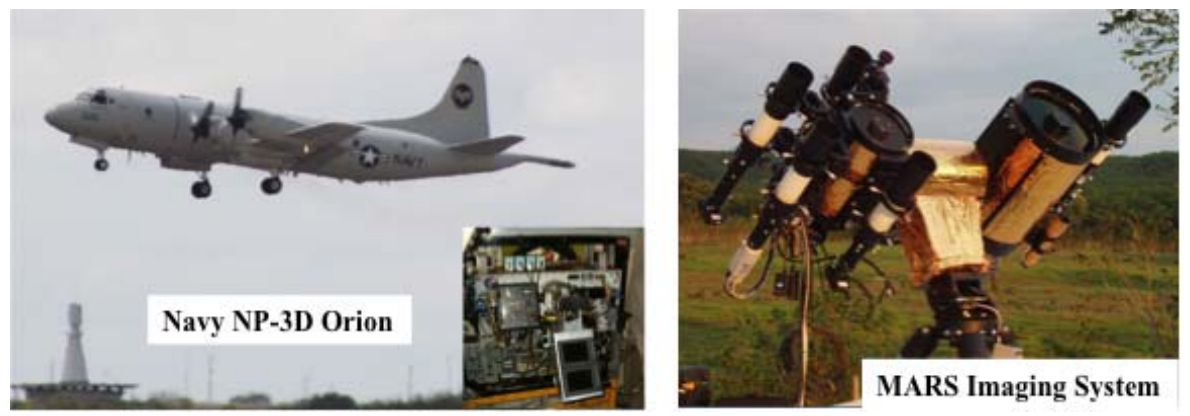

Figure 2. Imaging assets used for Orbiter re-entry data collects. 


\section{SENSOR DESCRIPTION AND CHARACTERIZATION}

The digital NIR camera used in both Cast Glance and MARS imaging systems is the Prosilica GC1380H. The camera pixel array size is $1360 \times 1024$ with a frame rate of $30 \mathrm{~Hz}$ at full resolution through a GigE interface. The camera's dynamic range is 12 bits. This wide dynamic range was valuable to minimize image saturation. The integration time can be varied from $10 \mu \mathrm{sec}$. to $60 \mathrm{sec}$. and is continuously variable in real time. The spectral response of the camera's CCD sensor is $0.4-1.05 \mu \mathrm{m}$. A long pass filter with cut on at $0.850 \mu \mathrm{m}$ is used to remove the spectral energy in the visible band. The acquired digital data file is stored in the flexible image transport system (FITS) format [18]. Frame acquisition time is stamped and the value is stored in the frame header. Custom data acquisition software was developed to control the camera's integration time in steps down to $10 \mu \mathrm{sec}$ [12]. The characterizations performed on the digital NIR system included radiometric, spatial, and spectral measurements using blackbody radiation sources and known targets. These characterizations were used as input to the radiance model to predict the imaged NIR radiometric response of the Orbiter's windward surface and allow for proper selection of the digital camera integration time to minimize pixel saturation. The radiometric characterization was performed using calibrated blackbody radiation sources set at various temperatures during low ambient light (night time or indoor) conditions. Radiometric calibration setup of the Cast Glance aircraft, typical blackbody setup orientation, and MARS calibration setup are shown in Figures 3a, 3b, and 3c respectively. The process involves the calibration of the measured radiance counts to actual temperature values. The calibration was performed in a temperature range of interest (up to $2400{ }^{\circ} \mathrm{F}$ ) at specified sensor integration times. The results are calibration curves that can then be used to convert the measured pixel intensity to quantitative radiance values. The spatial characterization was performed during data collect conditions on selected stars. This technique allows for an estimation of the spatial response imaging system. The spatial point spread function (PSF) or blurring is modeled as a Gaussian function. Predetermined stars such as Sirius were observed to estimate the PSF. A spectrometer was used to determine the imaging system's spectral response function. The spectral measurement was numerically combined with the cameras spectral response curve to determine the overall imaging system spectral response [13].

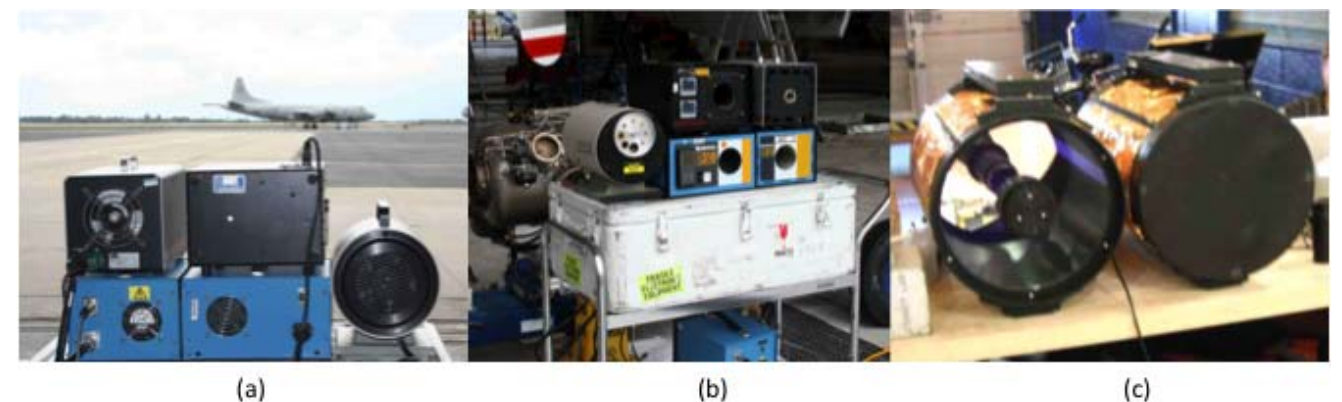

(a)

(b)

(c)

Figure 3. Imaging assets during radiometric calibration.

\section{NIR IMAGERY ASSESSMENT}

After data collection an initial assessment of the data is made. This assessment of the quality of the data collected includes number of saturated pixels, dropped frames, integration time adjustments vs. image number, when acquisition of signal was acquired, and loss of signal. This analysis involves the entire sequence of images acquired around 10,000 to 13,000 frames. Imagery of interest captured, such as reaction control system (RCS) firings, asymmetric boundary layer transition (ABLT), DTO BLT, contrail events, and best resolution images around the point of closest approach (PCA) are selected. A typical assessment is shown in Figure 4 for the HYTHIRM STS-125 mission where PCA was around 36.5 nautical miles (NM) and Orbiter's speed was Mach 15. As can be seen there are few saturated pixels. These images are a significant improvement over images shown in Figure 1.

\subsection{Image Histogram Analysis}

A more detailed assessment of the collected data is provided using a histogram analysis technique. The image histogram analysis method was used to analyze the NIR data frame by frame to determine the best resolution images by estimating 

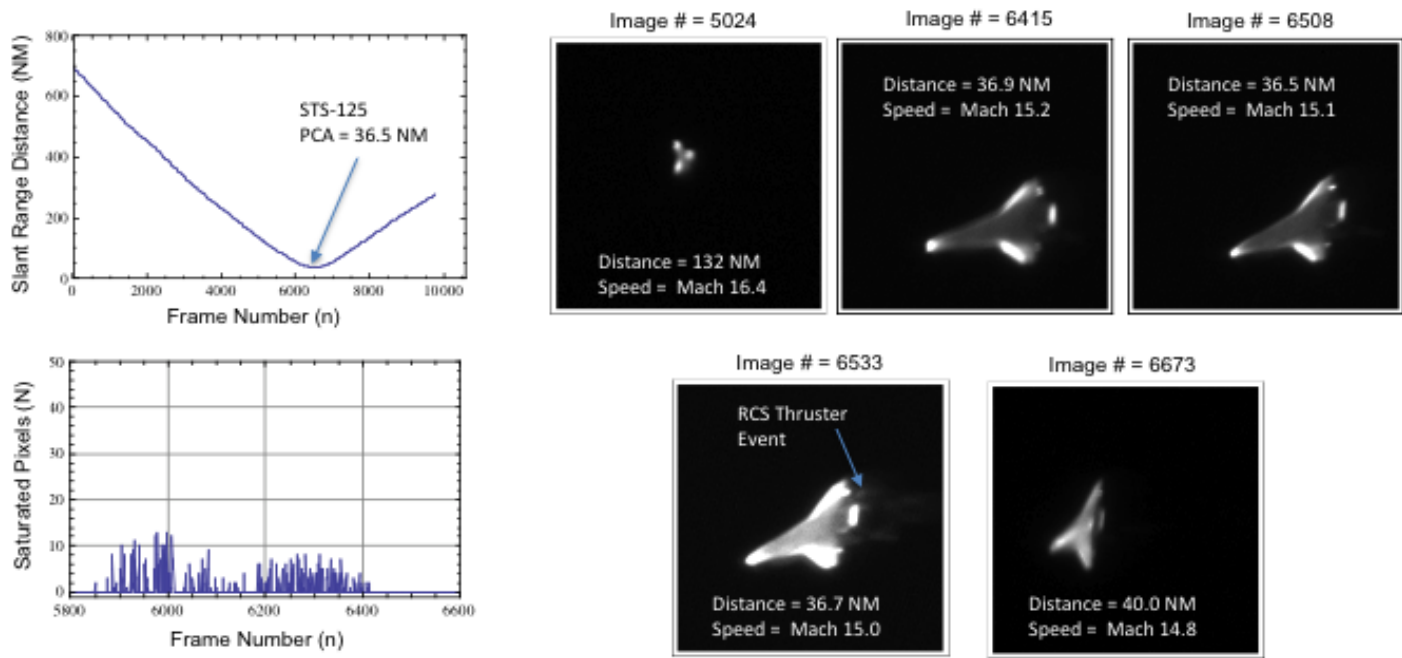

Image \# = 6533

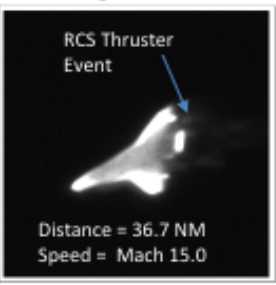

Image \# = 6673

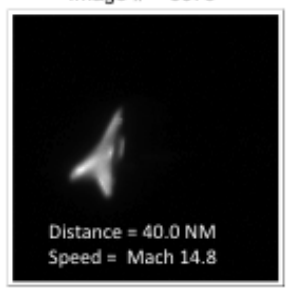

Figure 4. Imaging results for STS-125 HYTHIRM data collect.

the number of pixels on target. The number of pixels on target is determined by summing the pixels with counts above a threshold for each acquired frame. The equation is given as:

$$
N=\sum_{i=k}^{4095} \text { Bincounts }_{i}
$$

where $i$ is the counts, $k$ is the counts threshold value, and $N$ is the estimated Obriter's pixel resolution. Because the sky background is cool, compared to the Orbiter's windward surface which is hot (higher pixel counts), the number of pixels can be estimated by summing all the pixels associated with a value greater than approximately 3-5 times the average background counts. This is shown in Figure 5 where the peak resolution is compared to the slant range distance. The peak resolution image occurs at image \# 6257 not at image \# 6380 (image at minimum slant range distance). This indicates that at minimum slant range distance, the view angle to the windward surface is not optimal.
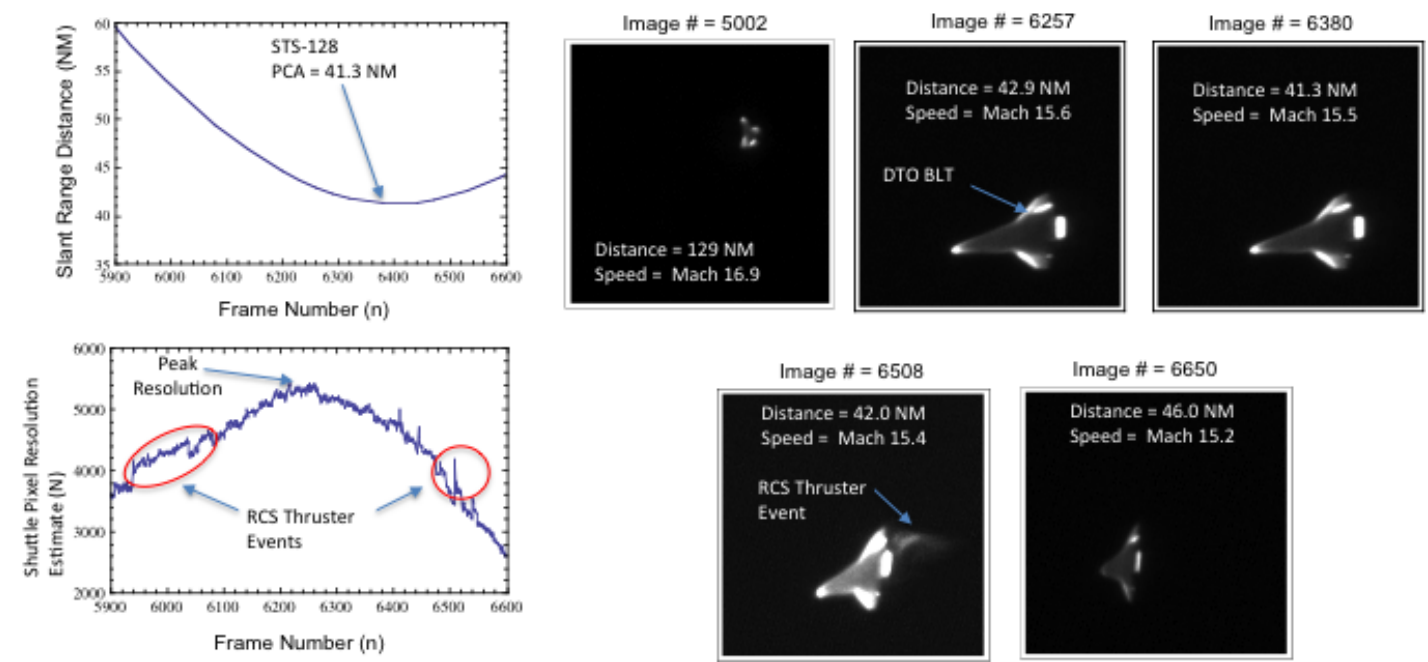

Figure 5. Imaging results for STS-128 HYTHIRM data collect with Orbiter pixel resolution estimate. 
In addition, the image histogram analysis method was used to determine acquisition and loss of signal, RCS firings, and signal loss to vignetting distortion. Vignetting distortion occurs when the optical pointing system is looking up near the edge of a window. The optical system is located permanently on the aircraft's starboard side. A combination of the STS 132 ground track, banking angle during decent and location of the Sun during acquisition required the positioning of the Cast Glance aircraft to be on the non-optimal side of the Orbiter re-entry track. Although the aircraft bank angle and gimbaled pointing system can help to compensate for vignetting it could not be avoided for this mission. Vignetting causes a reduction in image brightness, in extreme cases spatial distortions, and ultimately loss of signal. This is shown in Figure 6. The image histogram analysis curve was used to determine signal loss due to vignetting and therefore provides a technique to avoid imagery with vignetting loss.
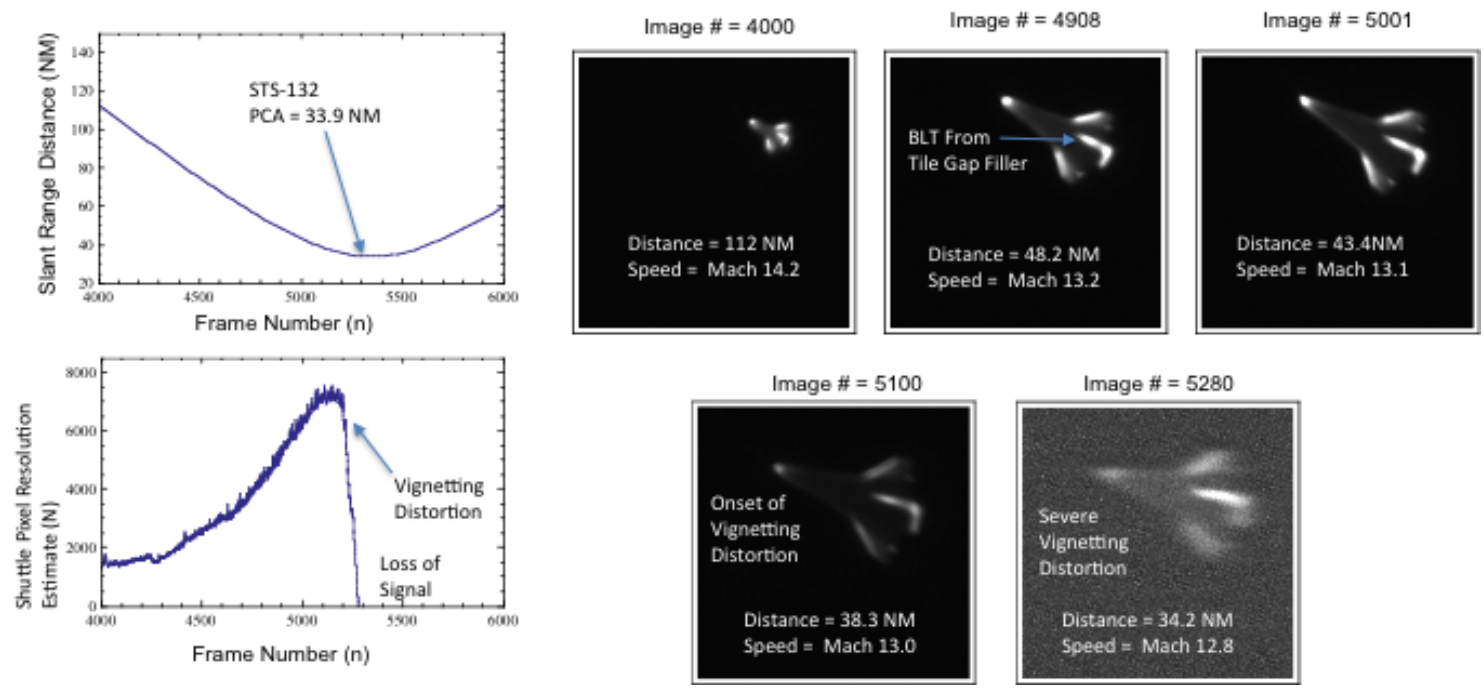

Figure 6. STS-132 HYTHIRM data collect with pixel resolution estimate showing vignetting distortion.

\subsection{Image Sharpness Measurement using Eigenvalue Decomposition}

STS-134 data collect provided never seen before imagery of the development and movement of a hypersonic boundary layer transition front at unprecedented high resolution (approximately $10.2 \mathrm{~cm}$ per pixel) [19]. This data was collected with the land based MARS imager. The combination of the Orbiter traveling at a high rate of speed along with atmospheric attenuation and tracking jitter can cause varying degrees of frame-to-frame blurring. The image histogram analysis is able to determine an optimal range, when the best resolution images are collected. However, it is not able to determine the amount of frame-to-frame blurring. The problem is that a sharper image would contain more bright pixel values thereby increasing the number of pixels above the threshold, however the collected light would be spread over fewer pixels. Similarly, a blurred image would increase the number of pixels above the threshold, because the light would be spread over more pixels, however the intensity would decrease thus lowering the total pixels. Image selection based on sharpness was done previously using a feature-based approach where the wing leading edges, nose, and body flap were used to determine sharpness [20,21]. Because STS-134 data collect provided imagery at a much higher resolution, a eigenvalue value decomposition technique was used to determine sharpness. Image sharpness using singular values provides a statistical approach to measure sharpness [15]. This technique is applicable for Orbiter imagery because the blur function or PSF contributes to the low spatial frequencies and the details of interest are in the higher spatial frequencies. The singular value decomposition is given below in Eq. 2.

$$
A=U \Lambda V^{T}
$$

Where $A$ is the image matrix which is real (image counts) and has dimension $\mathrm{m} \mathrm{x}(\mathrm{m}>\mathrm{n}), U$ contains the left singular vectors, $\Lambda$ is the diagonal matrix containing the singular values of $A$ arranged in decreasing order, and $V$ contains the 
right singular vectors. The sharpness metric $S$ is then given below in Eq. 3 [15].

$$
S=\operatorname{trace}\left[\Lambda_{k}\right]=\sum_{i=1}^{k} a_{i i}
$$

Where $a_{i i}$ are the diagonal singular values and $S$ is the sum of singular values up through $k$. The value $k$ is determined by reconstructing the image matrix $A$ where sufficient spatial information is restored for a representative sharp image. This is shown in Figure 7 for a sharp image collected during STS-134 re-entry showing thermal gradients or "fingers" associated with turbulent flow on the port wing. These fine details allow for further study to determine the origin of theses gradients [19]. The image reconstruction is given in Eq. 4 where $A_{k}$ is the reconstructed image and $u_{i}$ and $v_{i}$ are the left and right singular column vectors

$$
A_{k}=\sum_{i=1}^{k} a_{\mathrm{ii}}\left(u_{i} \cdot v_{i}^{T}\right)
$$

respectively corresponding to the singular values $a_{i i}$. Because the diagonal matrix $\Lambda$ contains the singular values in decreasing order most of the image information is contained up to $k=50$ as shown in Figure 7 with the line plots showing most of the detail is recaptured. For each acquired image, the eigenvalues are determined and the sharpness $S$ is calculated using Eq. 3. An example plot is shown in Figure 8 for the STS-134 data. Figure 8 shows the frame-by-frame variation in the image sharpness due to the blurring as previously discussed. Approximately two-thirds or less of the collected images are typically not selected or considered for subsequent processing [20]. Another benefit of this technique is viewing a series of images. When observing a series of images, blurring can distort the observation of interest such as the formation and movement of a boundary layer front. By using the sharpness metric, the undistorted sharp images in the sequence of interest can be selected for review temporally.
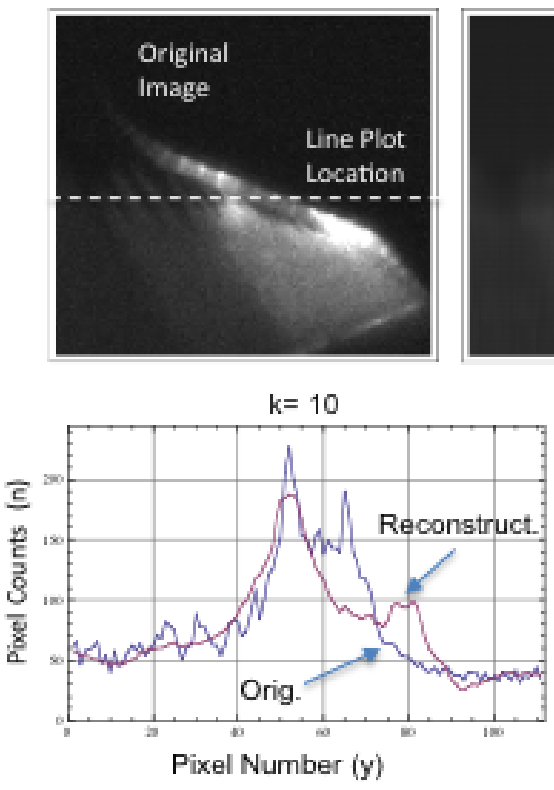
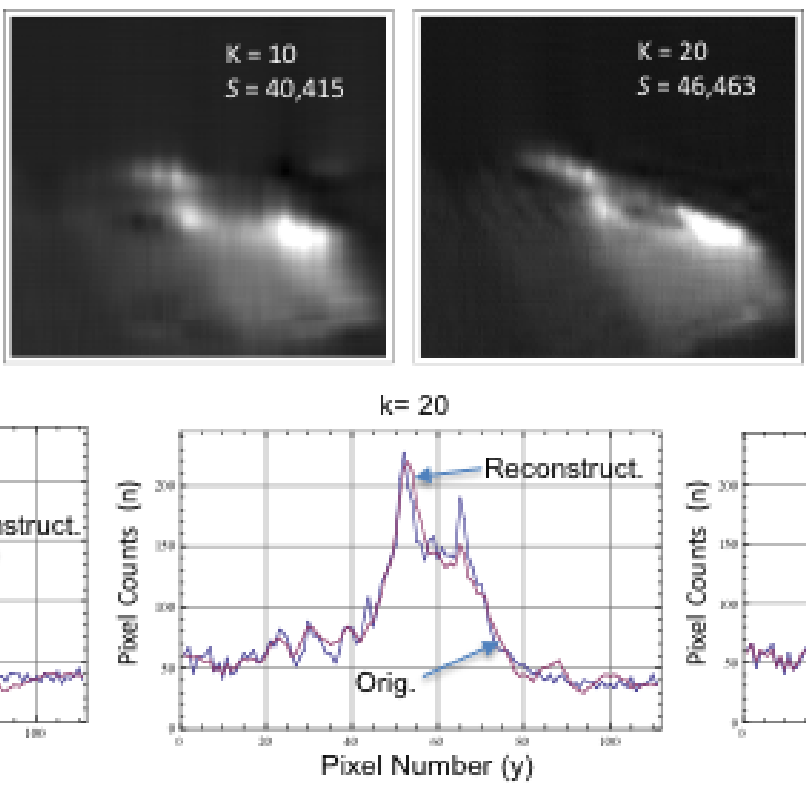
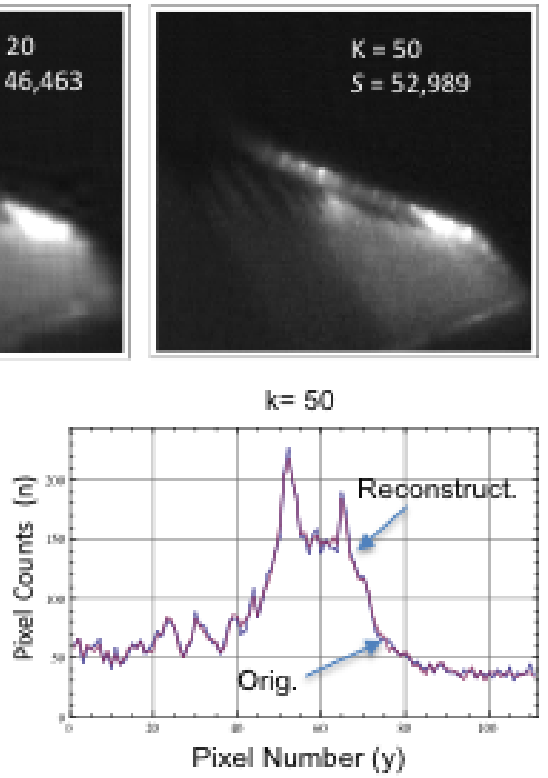

Figure 7. STS-134 imagery reconstructed to determine $k$ value for sharpness metric with line plot comparison.

\subsection{Optimal Image Selection using Image Histogram Analysis and Image Sharpness Measurement}

Combining the image histogram analysis technique with the image sharpness metric allows for the selection of the optimal images in the data collected. The image histogram technique determines the range of images where the optimal resolution or most pixels on the windward surface of the Orbiter is observed. Within that range the sharpest image or images can then be determined quantitatively using the eigenvalue technique. The optimal images for STS-119, STS125, STS-128, STS-131, STS-132, STS-133, and STS-134 missions are presented in Table 1 and shown in Figure 9. 


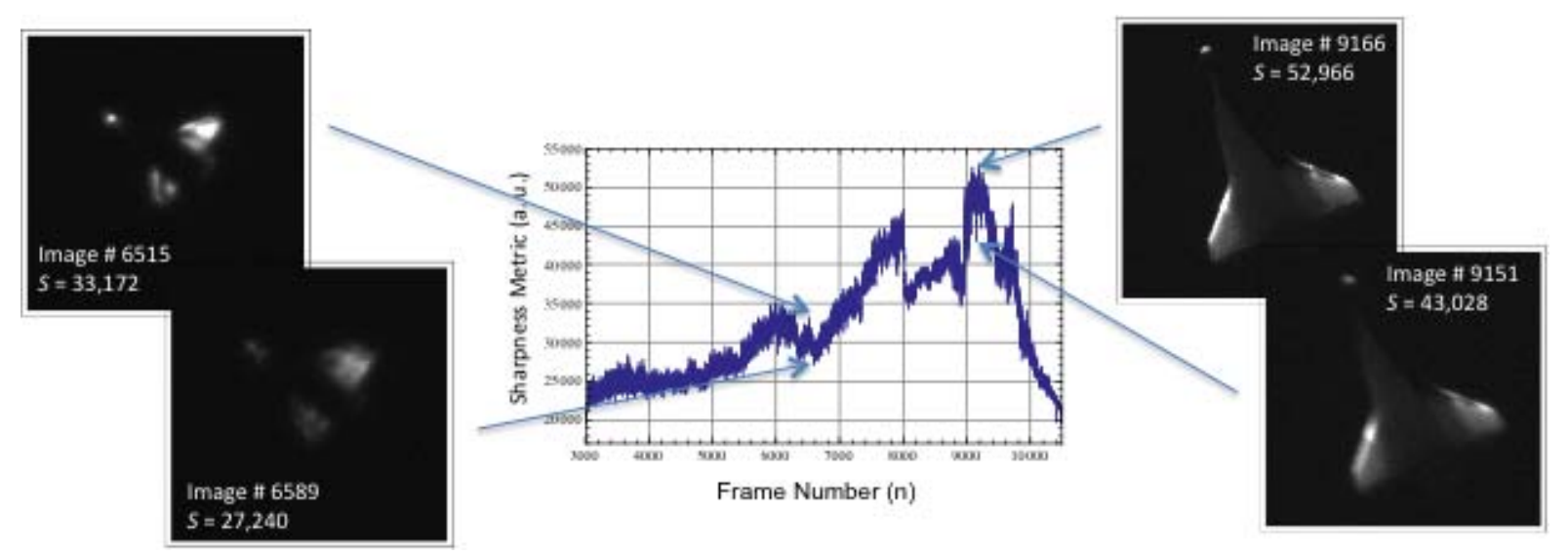

Figure 8. Plot of sharpness metric for STS-134 data with example images.

Table 1. Optimal image selection.

\begin{tabular}{|c|c|c|c|c|c|}
\hline Mission (Date) & $\begin{array}{l}\text { Imaging } \\
\text { Asset }\end{array}$ & $\begin{array}{l}\text { Maximum Resolution } \\
\text { Image Number Range }\end{array}$ & $\begin{array}{l}\text { Selected Sharp } \\
\text { Image Number }\end{array}$ & $\begin{array}{l}\text { Distance } \\
\text { (NM) }\end{array}$ & Mach \\
\hline STS-119 (3/28/2009) & Cast Glance & $3550-3750$ & 3562 & 30.6 & 8.5 \\
\hline STS-125 (5/24/2009) & Cast Glance & $6300-6500$ & 6391 & 38.0 & 14.3 \\
\hline STS-128 (9/12/2009) & Cast Glance & $6100-6400$ & 6127 & 46.8 & 14.8 \\
\hline STS-131 (5/26/2009) & MARS & $2622-2664$ & 2643 & 67.4 & 15.1 \\
\hline STS-132 (5/26/2010) & Cast Glance & $4900-5300$ & 4954 & 44.9 & 13.1 \\
\hline STS-133 (3/09/2011) & Cast Glance & $6900-7200$ & 6980 & 49.9 & 17.8 \\
\hline STS-134 (3/28/2011) & MARS & $8980-9300$ & 9166 & 29.6 & 5.7 \\
\hline
\end{tabular}
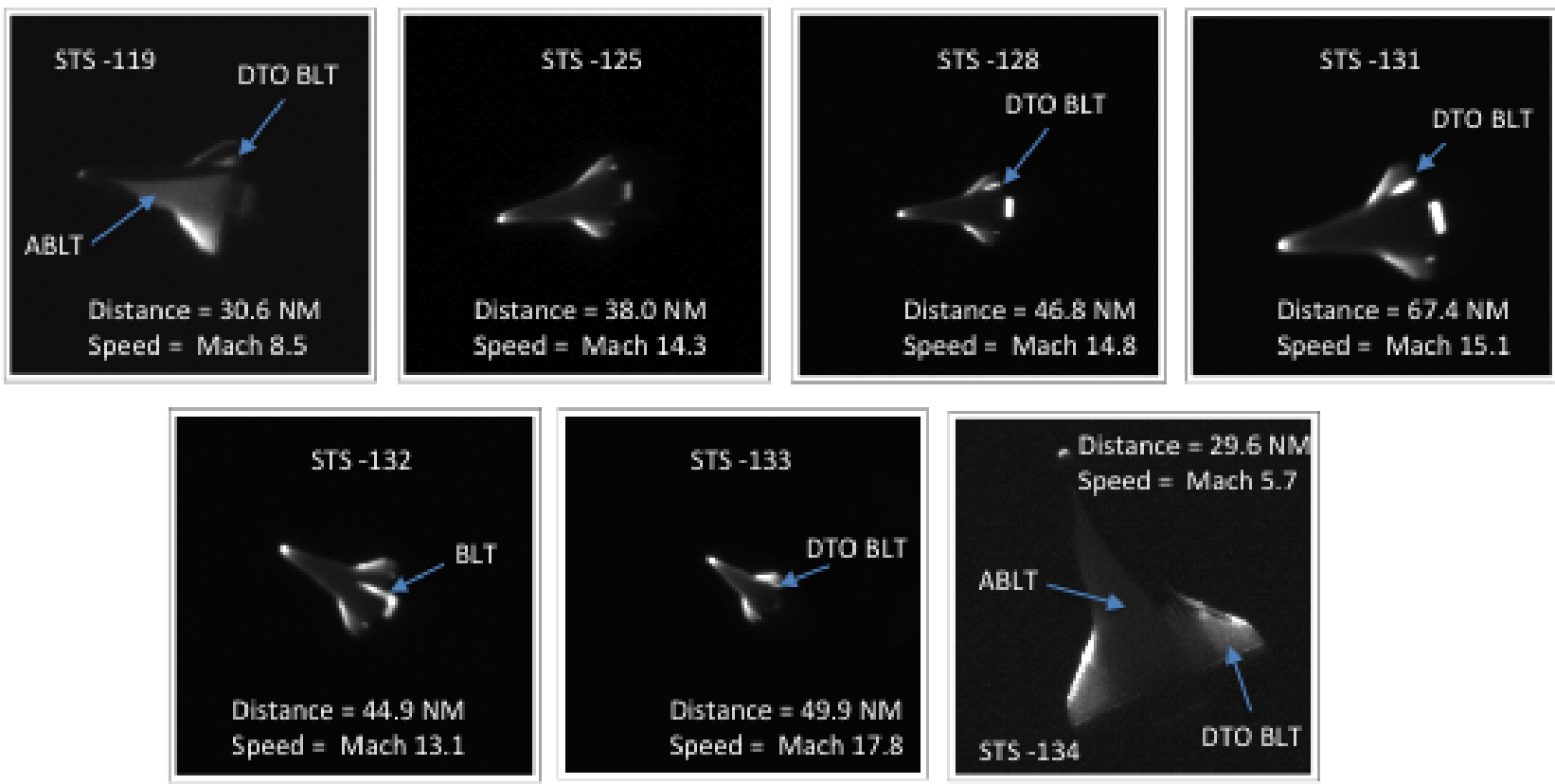

Figure 9. Selected optimal images for STS-119, STS-125, STS-128, STS-131, STS-132, STS-133, and STS-134. 


\section{IMAGE PROCESSING}

\subsection{Conversion to Temperature}

The process used to convert the raw image counts to quantitative temperature is shown schematically in Fig. 10. Image processing required selection of a series of frames for averaging (6 to 25 frames). Proper registration of multiple frames for averaging requires that the Orbiter orientation, aspect ratio, and optical blurring must be minimal within these frames. The selection of frames for averaging utilized a feature based approach as described by Spisz [20,21]. The results are similar to the sharpness metric discussed in this paper. A radiometric calibration curve is used to covert counts to a radiance image for a given integration time. The radiance is corrected for atmospheric path transmission, solar scattering from aerosols, and path radiance to produce the in-band radiance at the Orbiter's windward surface using MODTRAN atmospheric modeling. The object radiance was converted to temperature by using Planck's black body radiation law and the estimated in-band averaged reaction cured glass (RCG) coated tile emissivity value of 0.65 [22].

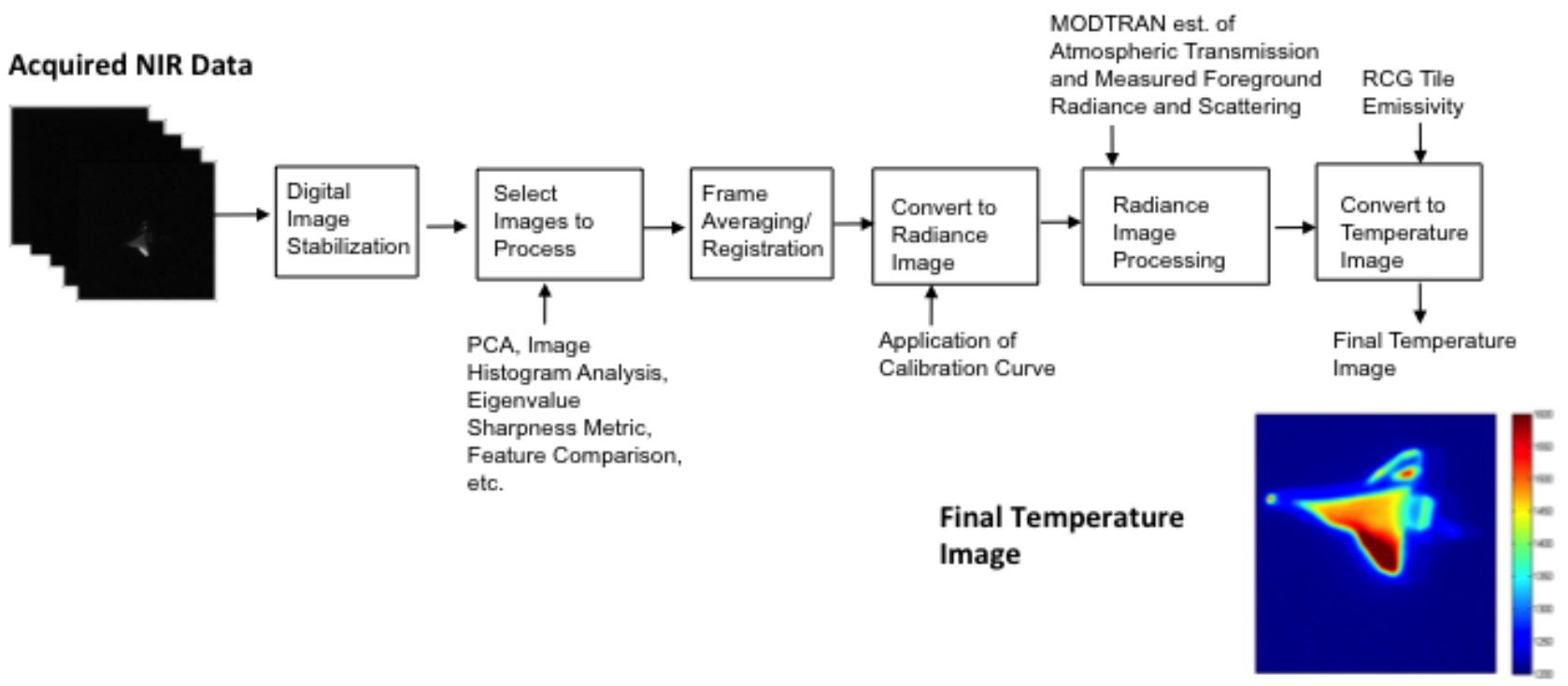

Figure 10. Conversion of image counts to temperature image.

\subsection{Comparison to Onboard Thermocouples}

Thermal imagery obtained from the HYTHIRM missions is compared to the Orbiters' onboard thermocouples values for STS-132, STS-133, and STS-134 in Figures 11a, 12a and 13a respectively. The comparisons were performed on the thermocouples located along the centerline. The actual locations of the thermocouples are in 3-dimensional space due to the curvature of the windward surface and using the centerline simplifies location of the thermocouples. The averaged magnitude difference error was $125^{\circ} \mathrm{F}$ for STS-132, STS-133, and STS-134 images and the thermocouple measurements were typically lower. These results are similar to previous errors obtained during STS-119, STS-125, and STS 128 comparisons $[12,20]$. The thermocouple offset error is $20^{\circ} \mathrm{F}$ cooler than the actual surface with uncertainties around +/$20^{\circ} \mathrm{F}$ [23], however there was significant scatter as shown in Figure 11b. Over a region of laminar flow (temperatures should be uniformly decreasing spatially from nose to aft) the scatter in thermocouple measurements suggests some additional thermocouple offset errors that have not been accounted for. A more rigorous mapping technique allowing for 3-Dimensional thermography has been developed [24]. This will allow for the exact determination of the thermocouple locations, especially those away from the centerline. Lastly, as expected, the measurement of lower temperatures (around $1,200{ }^{\circ} \mathrm{F}$ and below) will produce greater relative errors mainly due to the reduced long wave quantum efficiency of the CCD detector. 


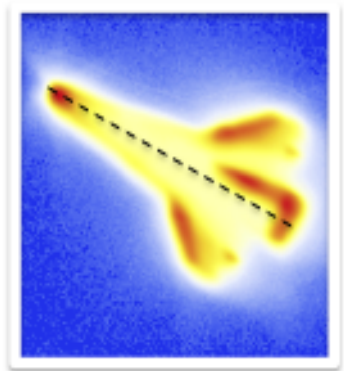

(a)

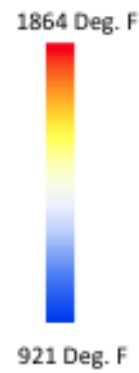

921 Deg. F

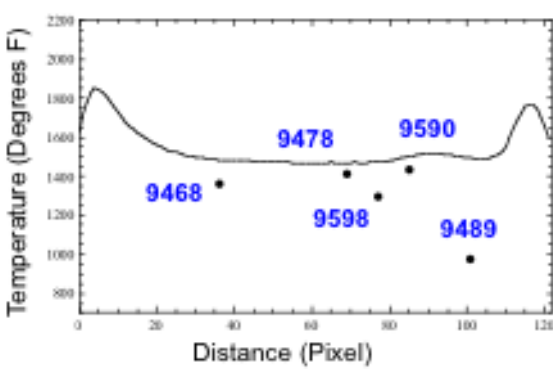

(b)

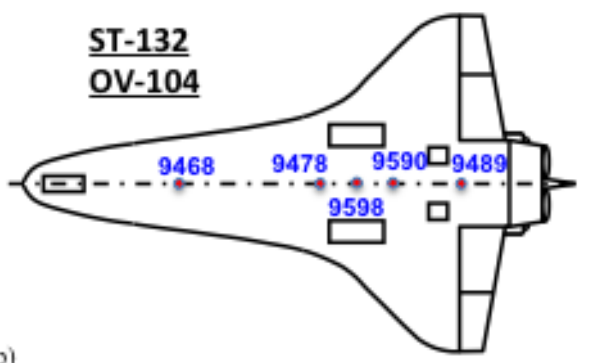

Figure 11. STS-132 centerline temperature comparison to thermocouple data.
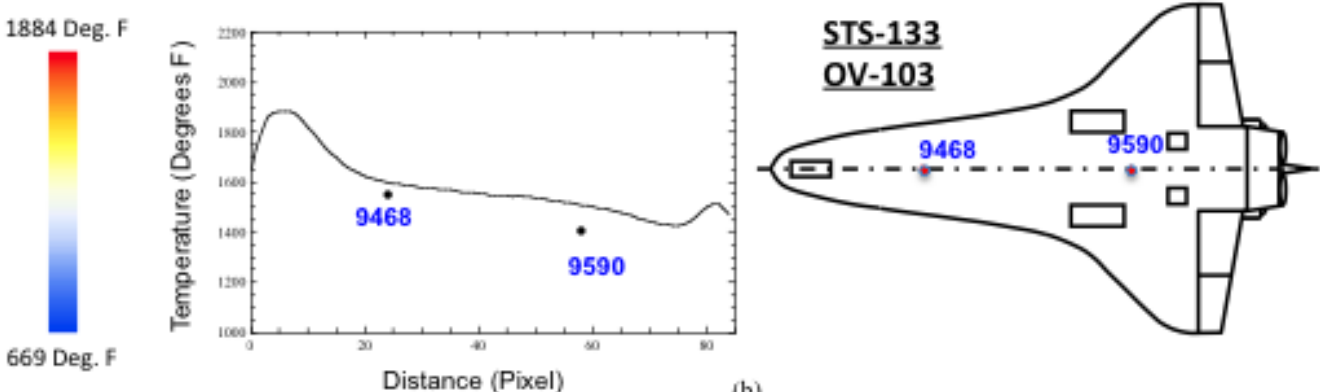

(b)

Figure 12. STS-133 centerline temperature comparison to thermocouple data.

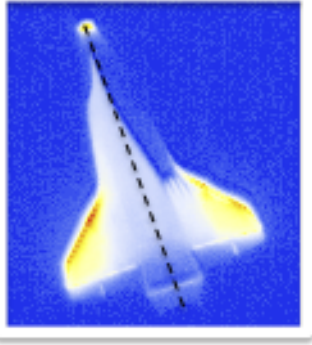

(a)

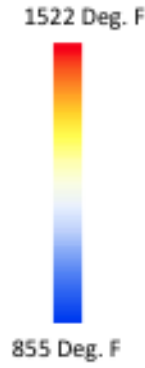

Figure 13. STS-134 centerline temperature comparison to thermocouple data.

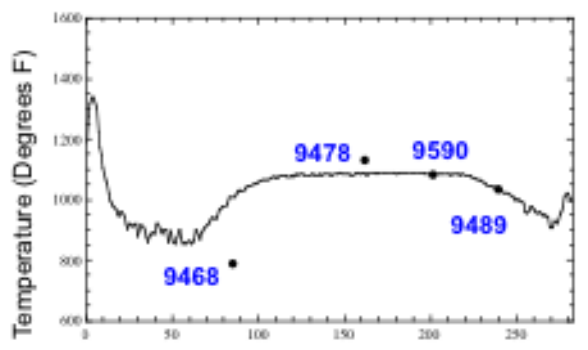

Distance (Pixel)

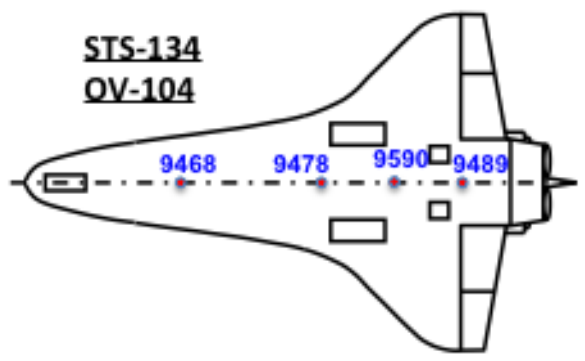

(b)

\section{CONCLUSIONS}

A method to analyze digital NIR imagery of a vehicle during hypersonic re-entry is presented. A histogram method is used to analyze the thermal imagery and is used to determine image saturation, frame dropouts and signal loss due to vignetting. The histogram analysis technique also determines the range of images during maximum pixel resolution. In addition, a technique to measure image sharpness using an eigenvalue decomposition approach is presented. The combination provided an automated method to select the optimal images. Lastly as shown in Figure 9 the image quality has improved greatly from the early mission data collects of STS-121 and STS-115 (Figure 1). The STS-134 data collect represents unprecedented spatial resolution that defines the future potential for remote quantitative thermography imaging for hypersonic research [25]. This will allow for development of future space vehicle designs. 


\section{REFERENCES}

[1] Wood, W. A., Kleb, W. L., Tang, C. Y., Palmer, G. E., Hyatt, A. J., Wise, A. J., McCloud, P. L., “Comparison of CFD Predictions with Shuttle Global Flight Thermal Imagery and Discrete Surface Measurements," AIAA Paper 2010-454, Jan. 2010.

[2] Horvath, T., Berry, S., Alter, S., Blanchard, R., Schwartz, R., Ross, M., and Tack, S., "Shuttle Entry Imaging Using Infrared Thermography,” AIAA-2007-4267, June 2007.

[3] Berry, S., Horvath, T., Schwartz, R., Ross, M., Campbell, C., and Anderson, B., "IR Imaging of Boundary Layer Transition Flight Experiments,” AIAA-2008-4026, June 2008.

[4] Ross, M., Werner, M., Mazuk, S., Blanchard, R., Horvath, T., Berry, S., Wood, W., and Schwartz, R., "Infrared Imagery of the Space Shuttle at Hypersonic Entry Conditions,” AIAA-2008-0636, 46th AIAA Aerospace Sciences Meeting and Exhibit, Reno, NV, Jan. 7-10, 2008.

[5] Horvath, T., Berry, S., Splinter, S., Daryabeigi, K., Wood, W., Schwartz, R., and Ross, M., "Assessment and Mission Planning Capability For Quantitative Aerothermodynamics Flight Measurements Using Remote Imaging," AIAA-2008-4022, June 2008.

[6] Splinter, S., Daryabeigi, K., Horvath, T., Mercer, C.D., Ghanbari, C. M., Ross, M. N., Tietjen, A., B., and Schwartz, R., J., "Solar Tower Experiments for Radiometric Calibration and Validation of Infrared Imaging Assets and Analysis Tools for Entry Aero-Heating Measurements,” AIAA-2008-4025, June 2008.

[7] Tietjen, A. B. and Hand, D. "Calibration and Data Analysis Summary Report-HYTHIRM Solar Tower Test," HYTHIRM Internal Report, Sept. 2008.

[8] Horvath, T. J., Tomek, D. M., Berger, K. T., Splinter, S. C, Zalameda, J. N., Krasa, P. W., Tack, S., Schwartz, R. J., Gibson, D. M., and Tietjen A. B., "The HYTHIRM Project: Flight Thermography of the Space Shuttle during Hypersonic Re-entry," AIAA Paper 2010-241, Jan. 2010.

[9] Tack, S., Tomek, D. M., Horvath, T. J., Verstynen, H. A., and Shea, E. J., "Cast Glance Near Infrared Imaging Observations of the Space Shuttle during Hypersonic Re-entry," AIAA Paper 2010-243, Jan. 2010.

[10] Gibson, D. M., Spisz, T. S., Taylor, J. C., Zalameda, J. N., Horvath, T. J., Tomek, D. M., Tietjen, A. B., Tack, S., and Bush, B. C., "HYTHIRM Radiance Modeling and Image Analyses in Support of STS-119, STS-125, and STS128 Space Shuttle Hypersonic Re-entries," AIAA Paper 2010-244, Jan. 2010.

[11]Berger, K. T., Anderson, B., Campbell, C., Garske, M., Saucedo, M. Kinder, G., Micklos, A., "Boundary Layer Transition Flight Experiment Overview,” AIAA Paper 2011-3323, June 2011.

[12] Zalameda, J. N., Horvath, T. J., Tomek, D. M., Tietjen, A. B., Gibson, D. M., Taylor, J. C., Tack, S., Bush, B. C., Mercer, C. D., and Shea, E. J., "Application of a Near Infrared Imaging System for Thermographic Imaging of the Space Shuttle during Hypersonic Re-entry," AIAA Paper 2010-245, Jan. 2010.

[13] Tietjen, A. B. and Hand, D. "Calibration and Data Analysis Summary Report-HYTHIRM STS-119, STS-125 and STS-128," HYTHIRM Internal Report, Dec. 2009.

[14] Andrews H. C. and Patterson C. L., "Singular Value Decomposition (SVD) Image Coding", IEEE Transactions on Communications, Volume 24 Issue 4, April 1976.

[15]Wee C. Y. and Paramesran R., "Image Sharpness Measure Using Eigenvalues", $9^{\text {th }}$ International Conference on Signal Processing, ICSP Oct. 2008.

[16] A. Berk, L.S. Bernstein, and D.C. Robertson, "MODTRAN: A moderate resolution model for LOWTRAN7", Report GL-TR-89-0122, Air Force Geophysics Lab., Bedford, MA, 1989.

[17] Rybicki G. B. and Lightman, A. P., "Radiative Processes in Astrophysics, New York: John Wiley and Sons, ISBN 0-471-82759-2, 1979.

[18] Hanisch, R. J., Farris, A., Greisen, E. W., Pence, W. D., Schlesinger, B. M., Teuben, P. J., Thompson, R. W., and Warnock, A., Definition of Flexible Image Transport (FITS), Astronomy \& Astrophysics, 376, 359-380, 2001.

[19] Horvath, T. J., Zalameda, J. J., Wood, W. A., Berry, S. A., Swartz, R. J., Dantowitz, R. F., Spisz, T. S., and Taylor, J. C., "Time Resolved Global Infrared Observations of Roughness Induced Boundary Layer Transition on the Space Shuttle Orbiter During STS-134 Reentry,” NATO RTO-MP-AVT-200-27, Applied Vehicle Technology Panel and Specialist's meeting on Hypersonic Laminar-Turbulent Transition, San Diego, CA, April 16-19, 2012.

[20] Spisz, T. S., Taylor, J. C., Gibson, D. M., Kwame, O. W., Horvath, T. J., Zalameda, J. N., Tomek, D. M., Berger, Tietjen, A. B., Tack, S., and Schwartz, R. J., "Processing Near-Infrared Imagery of Hypersonic Space Shuttle Reentries," Thermosense XXXII Conference at 2010 SPIE Defense, Security, and Sensing Symposium, 5-9 April 2010, Orlando, FL, Paper 7661-17. 
[21] Spisz, T. S., Taylor, J. C., Gibson, D. M., Kennerly, S. W., Kwame, O., Horvath, T. J., Zalameda, J. N., Kerns, R. V.,Shea, E. J., Mercer, C. D., Schwartz, R. J., Dantowitz, R. F., Kozubal, M. J., "Processing Ground-Based NearInfrared Imagery of Space Shuttle Reentries," Thermosense XXXIV Conference at 2012 SPIE Defense, Security, and Sensing Symposium, 23-27 April 2012, Baltimore, MD, Paper 8354-15.

[22] Bouslog, S. A. and Cunnington, G. R., "Emittance Measurements of RCG Coated Shuttle Tiles", AIAA Paper 920851, Jan. 1992. Wood, W. A., Kleb, W. L., Tang, C. Y., Palmer, G. E., Hyatt, A. J., Wise, A. J., and McCloud, P. L., "Comparison of CFD Predictions with Shuttle Global Flight Thermal Imagery and Discrete Surface Measurements," AIAA Paper 2010-454, Jan. 2010.

[23] Anderson, B., Campbell, C., Kinder, J., and Saucedo, L., "Boundary Layer Transition Flight Experiment Overview and In-Situ Measurements,” AIAA-2010-420, Jan. 2010.

[24] Taylor, J. C., Gibson, D. M., Spisz, T. S., and Horvath, T. J., “Global Thermography of the Space Shuttle during Hypersonic Re-entry," AIAA Paper 2011-3324, June 2011.

[25] Horvath, T. J., Kerns, R. V., Jones, K. M., Grinstead, J. H., Schwartz, R. J., Gibson, D. M., Taylor, J. C., Tack, S., and Dantowitz, R. F., "A Vision of Quantitative Imaging Technology for Validation of Advanced Flight Technologies,” AIAA-2011-3325, June 2011. 